\title{
Relación de la concentración de vitamina D en sangre con la masa muscular y la función cognitiva en mujeres posmenopáusicas
}

\author{
Sebastián Carranza-Lira, ${ }^{1 *}$ Melissa López-Chávez, ${ }^{2}$ Alejandra Díaz de León-de Luna, ${ }^{2}$ \\ Sergio Rosales-Ortiz ${ }^{3}$ y Guadalupe J. Méndez-González \\ ${ }^{1}$ División de Investigación en Salud; ${ }^{2}$ División de Educación en Salud; ${ }^{3}$ Servicio de Ginecología, ${ }^{4}$ Laboratorio Clínico. Unidad Médica de Alta \\ Especialidad, Hospital de Gineco Obstetricia Luis Castelazo Ayala, Instituto Mexicano del Seguro Social. Ciudad de México, México
}

\begin{abstract}
Resumen
Introducción: Las concentraciones bajas de vitamina $D$ se han asociado con la pérdida de masa muscular y la alteración de la función cognitiva. Objetivo: Conocer la relación de la concentración sanguínea de vitamina $D$ con la masa muscular y la función cognitiva en mujeres posmenopáusicas. Materiales y métodos: Se estudiaron 99 mujeres posmenopáusicas $\geq 50$ años. Se midió la circunferencia de la pantorrilla, los pliegues cutáneos tricipital, bicipital, subescapular y suprailíaco. Se calcularon: el área muscular del brazo, el área muscular libre de hueso y la masa muscular total. Se realizó la prueba corta de desempeño físico (PCDF), se aplicó el cuestionario de diagnóstico rápido de sarcopenia (SARC-F) y el Mini Examen del Estado Mental (MMSE). Se tomó una muestra de sangre para medir la concentración de vitamina $D$ en sangre. Para el análisis estadístico se utilizó la prueba $U$ de Mann-Whitney y análisis de correlación de Spearman. Resultados: Se encontró que a mayor edad hubieron mayores concentraciones de vitamina $D$ y mayor puntaje SARC- $F$. Las concentraciones de vitamina $D$ se correlacionaron negativamente con la fuerza de agarre, la PCDF y la puntuación total del MMSE. Conclusiones: La vitamina $D$ no tuvo una influencia positiva sobre la masa muscular. Se observó un mejor desempeño en el MMSE en aquellas con concentraciones más bajas de vitamina $D$.
\end{abstract}

PALABRAS CLAVE: Sarcopenia. Fuerza muscular. Posmenopausia. Vitamina D. Grasa corporal. Cognición.

\section{Relation of vitamin D blood concentration with muscle mass and cognitive function in postmenopausal women}

\begin{abstract}
Introduction: Low levels of vitamin D have been associated with muscle mass loss and cognitive function alteration. Objective: To find out the relationship of vitamin $D$ blood levels with muscle mass and cognitive function in postmenopausal women. Materials and methods: Ninety-nine postmenopausal women aged $\geq 50$ years were studied. Calf circumference, and tricipital, bicipital, subscapular and suprailiac skinfolds were measured. Arm muscle area, bone-free arm muscle area, and total muscle mass were calculated. The short physical performance battery (SPPB) was performed, and the sarcopenia rapid diagnostic questionnaire (SARC-F), as well as the Mini Mental State Examination (MMSE) were applied. A blood sample was taken to measure vitamin D blood concentration. For statistical analysis, Mann-Whitney's U-test and Spearman's
\end{abstract}

0016-3813/@ 2021 Academia Nacional de Medicina de México, A.C. Publicado por Permanyer. Este es un artículo open access bajo la licencia
Correspondencia:

*Sebastián Carranza Lira,

E-mail: drsebastiancarranza@gmail.com

Fecha de recepción: 08-07-2021

Fecha de aceptación: 12-08-2021 CC BY-NC-ND (http://creativecommons.org/licenses/by-nc-nd/4.0/).
Gac Med Mex. 2021;157:503-511

Disponible en PubMed

www.gacetamedicademexico.com 
correlation analysis were used. Results: It was found that, the older the age, the higher the vitamin D levels, as well as higher SARC-F score. Vitamin D levels were negatively correlated with grip strength SPP and the MMSE total score. There was a negative correlation between vitamin $D$ levels and MMSE global score. Conclusions: Vitamin $D$ did not have a positive influence on muscle mass. A better MMSE performance was observed in those with lower vitamin D levels.

KEY WORDS: Sarcopenia. Muscular strength. Post-menopause. Vitamin D. Body fat. Cognition.

\section{Introducción}

La sarcopenia se caracteriza por la pérdida lenta y progresiva de masa muscular ${ }^{1-3}$, con una prevalencia en mayores de 60 años del 3 al 52\%4,5. Para su diagnóstico es necesario determinar la masa muscular, la fuerza muscular y el rendimiento físico ${ }^{6}$. La masa muscular se puede medir por densitometría de doble emisión de rayos $X$ o mediciones antropométricas ${ }^{7}$. También existe un cuestionario para el diagnóstico rápido de sarcopenia (SARC-F) que permite excluir discapacidad muscular y sarcopenia ${ }^{8,9}$.

La función muscular se puede evaluar mediante la fuerza de agarre de la mano $0^{10,11}$, la capacidad funcional midiendo la velocidad de la marcha en una distancia de $10 \mathrm{~m}$ y el rendimiento por el tiempo en sentarse y pararse cinco veces ${ }^{7,8,12-14}$. La prueba corta de desempeño físico (PCDF) evalúa el equilibrio, el tiempo para caminar cuatro metros y el tiempo para pararse y sentarse cinco veces ${ }^{13-15}$.

Las concentraciones bajas de vitamina D [25(OH)D] se han asociado con cambios desfavorables en la masa muscular y el rendimiento físico ${ }^{16}$, una mayor pérdida de masa muscular ${ }^{17} \mathrm{y}$ con mayor masa grasa y deterioro del metabolismo de la glucosa independientemente del índice de masa corporal (IMC) ${ }^{18,19}$.

Se ha reportado que la menopausia antes de los 45 años está asociada con un mayor riesgo de sarcopenia, sin poder demostrar que los cambios hormonales posmenopáusicos modifiquen el deterioro muscular ${ }^{20}$.

La función cognitiva se ve afectada por la edad y el uso de la terapia hormonal ${ }^{21,22}$. La deficiencia de $25(\mathrm{OH}) \mathrm{D}$ disminuye el rendimiento en las pruebas de función cognitiva ${ }^{23-28}$, sin embargo, en un estudio mexicano no se encontró relación entre la $25(\mathrm{OH}) \mathrm{D}$ y el rendimiento cognitivo ${ }^{29}$ y en otro no se encontró relación con la demencia en hombres ${ }^{30}$.

Para el diagnóstico de deterioro cognitivo se ha utilizado el Mini Examen del Estado Mental (MMSE) ${ }^{31}$, el cual cuantifica el rendimiento cognitivo global y permite la estadificación de la demencia ${ }^{32}$.
El objetivo del presente trabajo fue conocer la relación entre la concentración de $25(\mathrm{OH}) \mathrm{D}$ en sangre con la masa muscular y la función cognitiva en mujeres posmenopáusicas.

\section{Material y métodos}

Se estudiaron mujeres posmenopáusicas de 50 años o más que acudieron a la unidad médica de alta especialidad. No se incluyeron aquellas con antecedentes de cáncer de mama, enfermedad cardiaca, enfermedad pulmonar obstructiva crónica, nefropatías, síndromes de malabsorción, reemplazo de cadera, osteoartritis, antecedentes de traumatismos que impidieran el caminar o el agarre manual.

En todas ellas se investigó: edad (años), educación, ocupación, tipo de trabajo, realización de deporte. También se documentaron antecedentes obstétricos, de diabetes mellitus, tiempo transcurrido desde la menopausia y uso de terapia hormonal de reemplazo.

En todas se determinó el peso $(\mathrm{kg})$, la talla $(\mathrm{m})$, la circunferencia de la cintura $(\mathrm{cm})$, la circunferencia de la cadera $(\mathrm{cm})$ y se calculó el índice de masa corporal (IMC, $\mathrm{kg} / \mathrm{m}^{2}$ ) y el índice cintura: cadera (ICC, circunferencia de la cintura: circunferencia de la cadera).

También se evaluó: circunferencia muscular del brazo $=$ circunferencia del brazo - (pliegue cutáneo tricipital $\mathrm{x} \pi$ ); área muscular del brazo $(\mathrm{AMB})=$ circunferencia muscular del brazo $2 / 4 \pi$. Área muscular del brazo libre de hueso $(\mathrm{AMBLH})=\mathrm{AMB}-6.5$; masa muscular total (MMT) (kg): (altura, cm) $(0.0264)+$ (0.0029 x AMBLH). Se midieron los pliegues cutáneos tricipital, bicipital, subescapular y suprailíaco $(\mathrm{mm})$ con un plicómetro Lange ${ }^{33-35}$.

El porcentaje de grasa se calculó después de haber medido los cuatro pliegues cutáneos (bicipital, tricipital, subescapular y suprailíaco) con la fórmula de Durnin \& Wormersley para estimar la densidad corporal en $\mathrm{kg} / \mathrm{l})$. La fórmula se seleccionó según la edad: donde $\mathrm{D}=$ densidad corporal $(\mathrm{g} / \mathrm{ml}), \mathrm{L}=$ logaritmo de la suma de los 4 pliegues cutáneos en $\mathrm{mm}$, para mujeres mayores de 50 años $D=1,1339-(0.0645 \times L)$, 
$\%$ grasa $=(495 /$ densidad corporal $)-450$. También se midió la circunferencia de la pantorrilla.

La fuerza de agarre se midió con un dinamómetro Camry Modelo EH101, se realizaron dos mediciones y se consideró el promedio de ellas para el análisis.

El equilibrio se evaluó en una escala de 0-4, considerando una calificación para la bipedestación con los pies juntos, con la punta del pie tocando el lado del talón y con la punta del pie tocando la parte posterior del talón. El tiempo para caminar 4 metros se evaluó en una escala de 0-4 según el tiempo que tomara en recorrerlos y el tiempo para pararse y sentarse cinco veces también en una escala de 0-4.

La PCDF considera bajo rendimiento cuando es 0-6 puntos, rendimiento intermedio cuando es 7-9 y alto rendimiento cuando es $10-12^{20}$.

Se realizó ecografía abdominal con un equipo Voluson 730 Pro (General Electric Healthcare, Austria $\mathrm{GM} \mathrm{GH}$ ), con un transductor de $3.5 \mathrm{MHz}$ para medir la grasa subcutánea y visceral (intraabdominal) ${ }^{19}$.

Se aplicó el SARC-F, el cual evalúa cinco parámetros: fuerza, asistencia para caminar, levantarse de una silla, subir escaleras y caídas, considerando una puntuación $\geq 4$ como predictiva de sarcopenia 8 .

Se aplicó el MMSE y se consideró demencia cuando la puntuación fue menor a 12 puntos, deterioro cognitivo cuando estaba entre 12 y 24 , deterioro cognitivo dudoso cuando 25 o 26 y normal cuando $\geq 27^{30}$.

La 25(OH)D se midió mediante ensayo de fijación electroquimioluminiscente $\left(\right.$ Cobas $\left.^{\circledR}\right)$, considerándose normal cuando $\geq 30 \mathrm{ng} / \mathrm{ml}$.

El cálculo del tamaño de la muestra fue como sigue: dado que había presupuesto para 100 pruebas y en los perfiles demográficos de las Naciones Unidas de la Comisión Económica para América Latina (CEPAL), en la que la población de 50-59 años corresponde al $28.7 \%$, de $60-6918.6 \%$, de $70-7910.6 \%$ y de 80 o más de $5.7 \%$, quedaron respectivamente para el grupo de 50-59 45 participantes, para el de 60-69 29, para el de 70-79 17 y para el de 80 o más, 9 participantes.

Análisis estadístico: dada la distribución de la muestra, la comparación entre los grupos se realizó con la prueba $U$ de Mann-Whitney. Se realizó análisis de correlación de Spearman entre las concentraciones de 25(OH)D y las evaluaciones para sarcopenia y el MMSE.

El protocolo fue autorizado por el Comité de Ética en Investigación y el Comité Local de Investigación en Salud con el n. ${ }^{\circ}$ de registro R-2019-3606-039 y las
Tabla 1. Datos generales en una población de 99 mujeres posmenopáusicas mayores de 50 años*

$\begin{array}{lc}\text { Edad (años) } & 60.0(50.0-90.0) \\ \text { Veces por semana que realiza actividad física } & 0.0(0-7) \\ \text { Peso }(\mathrm{kg}) & 66.0(43.1-99.5) \\ \text { Talla }(\mathrm{m}) & 1.6(1.4-1.7) \\ \text { Índice de masa corporal }\left(\mathrm{kg} / \mathrm{m}^{2}\right) & 27.4(18.6-41.8) \\ \text { Cintura }(\mathrm{cm}) & 86.5(66.9-120.0) \\ \text { Cadera }(\mathrm{cm}) & 102.0(78.0-132.0) \\ \text { Índice cintura:cadera } & 0.86(0.69-0.99) \\ \text { Tensión arterial sistólica }(\mathrm{mmHg}) & 120.0(95-150) \\ \text { Tensión arterial diastólica }(\mathrm{mmHg}) & 77.5(50-90)\end{array}$

*Los resultados expresan medianas (mínimos y máximos).

participantes firmaron la carta de consentimiento informado.

\section{Resultados}

Se incluyeron 100 mujeres posmenopáusicas, pero debido a que una muestra resultó dañada y no hubo recursos para procesarla nuevamente solo se estudiaron 99 mujeres

Los datos del grupo sin dividir se muestran en las tablas 1 y 2 .

Al dividir la población según la concentración de 25(OH)D, quedaron 3 grupos: grupo $1(0 \mathrm{a}<11 \mathrm{ng} / \mathrm{ml})$, grupo $2(11 \mathrm{a}<30 \mathrm{ng} / \mathrm{ml})$ y grupo $3(\geq 30 \mathrm{ng} / \mathrm{ml})$ (Tabla 3).

Se encontró que la fuerza de agarre fue significativamente menor en el grupo 3. La puntuación de la PCDF fue menor a mayor concentración de 25(OH)D. La proporción grasa visceral/grasa subcutánea fue menor en el grupo 2. La puntuación del SARC-F fue significativamente mayor en el grupo 3 (Tabla 3). Hubo una mayor proporción de mujeres con SARC-F normal (88.9\%) en el grupo con 25(OH)D entre 11 a $<30 \mathrm{ng} / \mathrm{ml}$ en comparación con los otros dos grupos $(p<0.006)$.

Al dividir la población por grupos de edad: grupo 1 (50 a < 60 años), grupo 2 (60 a $<70$ años), grupo 3 (70 a < 80 años) y grupo 4 ( $\geq 80$ años), se encontró que la $25(\mathrm{OH}) \mathrm{D}$ era mayor a mayor edad y la puntuación del MMSE menor (Tabla 4).

La fuerza de agarre y la puntuación de la PCDF fueron mayores en el grupo $<60$ años. La grasa 
Tabla 2. Concentraciones de vitamina D, marcadores de masa muscular, grasa visceral, desempeño funcional y MMSE en 99 pacientes posmenopáusicas mayores de 50 años ${ }^{\star}$

\begin{tabular}{lc}
\hline Vitamina D (ng/ml) & $17.2(3.5-37.1)$ \\
\hline Área muscular de brazo (cm²) & $28.7(16.3-68.3)$ \\
\hline Área muscular de brazo libre de hueso (cm²) & $22.2(10.1-61.8)$ \\
\hline Masa muscular total (kg) & $16.9(10.6-34.5)$ \\
\hline Densidad corporal (g/cm³) & 1.007 (0.994-1.039) \\
\hline Porcentaje de grasa (\%) & $41.6(26.6-48.1)$ \\
\hline Circunferencia del brazo (cm) & $27.0(16.0-35.0)$ \\
\hline Circunferencia de pantorrilla (cm) & $32.5(25.0-42.0)$ \\
\hline Promedio de fuerza de agarre (kg) & $19.8(4.1-39.6)$ \\
\hline Sentado-parado (s) & $10.7(4.9-38.3)$ \\
\hline Equilibrio pies juntos (s) & $30.0(10.5-30.0)$ \\
\hline Equilibrio resultadosstionario para diagnóstico rápido de sarcopenia; MMSE: Mini mental Scale \\
\hline Examination.
\end{tabular}
Examination.

visceral fue mayor en el grupo 2. La puntuación SARC- $F$ fue mayor en el grupo $\geq 80$ años.
La proporción de mujeres con SARC-F sugestivo de sarcopenia fue mayor en el grupo de mayor edad $(75 \%)(p<0.001)$ y la puntuación total del MMSE fue menor en el mismo grupo (Tabla 4).

Después de dividir el grupo según el IMC: grupo 1 $(\leq 27.0)$ y grupo 2 (> 27.0), se encontró que la densidad corporal y la puntuación de la PCDF fueron mayores en el grupo 1. La MMT, el porcentaje de grasa, la grasa subcutánea y visceral, las concentraciones de 25(OH)D y la puntuación del MMSE fueron más altas en el grupo con IMC > 27 (Tabla 5).

Después de dividir según el ICC: grupo $1(\leq 0.85)$ y grupo 2 (>0.85), se encontró que la densidad corporal fue mayor en el grupo 2 y el porcentaje de grasa, la fuerza de agarre y la suma de la PCDF fueron mayores en el grupo 1 (Tabla 5).

En el análisis de correlación de Spearman, hubo correlación entre la concentración de $25(\mathrm{OH}) \mathrm{D}$ con: la edad $0.443(p<0.001)$, la fuerza de agarre -0.242 $(p<0.016)$ y la puntuación de la PCDF de -0.590 $(p<0.001)$, puntuación del MMSE -0.228 ( $p<$ $0.023)$.

La edad correlacionó con: fuerza de agarre -0.497 $(p<0.001)$, puntaje de la PCDF $-0.683(p<0.001)$, puntaje del SARC-F $0.429(p<0.001)$ y puntuación del MMSE $-0.243(p<0.015)$.

La fuerza de agarre correlacionó con la puntuación de la PCDF 0.506 ( $p<0.001)$, la puntuación del SARC-F $-0.423(p<0.001)$ y la puntuación del MMSE $0.284(p<0.004)$.

La puntuación de la PCDF correlacionó con la puntuación del SARC-F $-0.587(p<0.001)$ y con la puntuación del MMSE $0.298(\mathrm{p}<0.003)$.

La masa muscular total correlacionó con el MMSE $0.282(p<0.005)$ (Tabla 6).

\section{Discusión}

En este estudio las concentraciones de $25(\mathrm{OH}) \mathrm{D}$ correlacionaron negativamente con algunos de los parámetros musculares (fuerza de agarre y puntuación de la PCDF), pero no con el puntaje SARC-F, lo que indica que la $25(\mathrm{OH}) \mathrm{D}$ no está relacionada con la sarcopenia ${ }^{16,17}$. Sin embargo, la edad influyó negativamente en los parámetros musculares, concordante con lo ya reportado ${ }^{35}$. Se observó que a mayor edad tenían mayor concentraciones de $25(\mathrm{OH}) \mathrm{D}$, para lo cual no tenemos explicación. Cabe mencionar que en este estudio solo cuatro mujeres presentaron $25(\mathrm{OH})$ $\mathrm{D}$ dentro de parámetros normales, de acuerdo con lo descrito en la literatura, donde hasta el $90 \%$ de los 
Tabla 3. Valores obtenidos en marcadores de masa muscular, grasa visceral, desempeño funcional y MMSE en 99 pacientes posmenopáusicas mayores de 50 años divididas según las concentraciones de vitamina $D$

\begin{tabular}{|c|c|c|c|}
\hline & $\begin{array}{c}0 a<11 \mathrm{ng} / \mathrm{ml} \\
(\mathrm{n}=11)\end{array}$ & $\begin{array}{c}11 \mathrm{a}<30 \mathrm{ng} / \mathrm{ml} \\
(\mathrm{n}=84)\end{array}$ & $\begin{array}{c}\geq 30 \mathrm{ng} / \mathrm{ml} \\
(\mathrm{n}=4)\end{array}$ \\
\hline Vitamina D (ng/ml) & $9.7(3.5-10.9)^{a, b}$ & $17.6(11.3-29.7)^{\mathrm{a}, \mathrm{c}}$ & $30.3(30.1-37.1)^{b, c}$ \\
\hline Edad (años) & $57.0(51.0-68.0)^{\mathrm{d}, \mathrm{e}}$ & $60.5(50.0-90.0)^{d, f}$ & $85.5(72.0-88.0)^{e, f}$ \\
\hline Peso (kg) & $63.5(48.6-9.50)$ & $66.5(43.1-90.1)$ & $71.5(55.0-82.0)$ \\
\hline Talla (m) & $1.56(1.47-1.68)$ & $1.54(1.42-1.70)$ & $1.56-1.40-1.69)$ \\
\hline Índice de masa corporal (kg/m²) & $25.9(20-39.9)$ & $27.5(18.6-36.4)$ & $29.4(19.3-41.8)$ \\
\hline Perímetro de la cintura $(\mathrm{cm})$ & $83.0(66.0-120.0)$ & $86.5(68.0-106.0)$ & $95.0(80.0-108.0)$ \\
\hline Perímetro de la cadera $(\mathrm{cm})$ & $96.0(68.0-132.0)$ & $102.0(82.0-121.0)$ & $109.50(105.0-112.0)$ \\
\hline Índice cintura cadera & $0.86(0.74-0.99)$ & $0.86(0.69-0.98)$ & $0.87(0.76-0.96)$ \\
\hline Presión arterial sistólica (mmHg) & $120(100-140)$ & $120(95-150)^{g}$ & $135(120-140)^{9}$ \\
\hline Presión arterial diastólica (mmHg) & $80(60-90)$ & $70(50-90)$ & $80(60-90)$ \\
\hline Área muscular de brazo & $31.2(25.5-38.5)$ & $28.1(16.6-68.3)$ & $31.0(19.0-51.6)$ \\
\hline Área muscular de brazo libre de hueso $\left(\mathrm{cm}^{2}\right)$ & $24.7(19.0-32.0)$ & $21.6(10.1-61.8)$ & $24.5(12.5-45.1)$ \\
\hline Masa muscular total (kg) & $18.2(15.7-20.9)$ & $16.7(10.6-34.5)$ & $17.7(13.8-26.4)$ \\
\hline Densidad corporal & $1.011(0.998-1.031)$ & $1.007(0.994-1.039)$ & $1.000(0.996-1.009)$ \\
\hline Porcentaje de grasa (\%) & $39.4(30.3-46.0)$ & $41.6(26.6-48.1)$ & $44.4(40.4-46.7)$ \\
\hline Promedio de fuerza de agarre $(\mathrm{kg})$ & $20.9(10.6-29.7)^{\mathrm{h}}$ & $19.90(4.1-39.6)^{i}$ & $11.6(8.8-16.7)^{\mathrm{h}, \mathrm{i}}$ \\
\hline Puntuación de la prueba corta de desempeño físico & $12.0(6.0-12.0)^{j, k}$ & $11.0(2.0-12.0)^{j .1}$ & $3.0(2.0-4.0)^{\mathrm{k} .1}$ \\
\hline Grasa subcutánea (cm) & $1.6(1.0-4.9)$ & $2.0(0.7-5.3)$ & $3.5(1.2-3.8)$ \\
\hline Grasa visceral (cm) & $4.9(2.6-8.9)$ & $4.1(1.4-8.5)$ & $4.2(3.0-6.5)$ \\
\hline Grasa visceral/subcutánea & $2.1(1.8-5.0)^{\mathrm{m}}$ & $1.8(0.9-5.8)^{m}$ & $1.6(0.8-3.0)$ \\
\hline SARC-F & $0.0(0.0-6.0)^{n}$ & $1.0(0.0-11.0)^{\circ}$ & $8.0(0.0-10.0)^{n, o}$ \\
\hline MMSE orientación temporal & $5.0(3.0-5.0)$ & $5.0(2.0-5.0)$ & $5.0(5.0-5.0)$ \\
\hline MMSE orientación espacial & $5.0(3.0-6.0)$ & $5.0(3.0-5.0)$ & $5.0(4.0-5.0)$ \\
\hline MMSE recuerdo inmediato & $1.0(1.0-1.0)$ & $1.0(1.0-1.0)$ & $1.0(1.0-1.0)$ \\
\hline MMSE fijación & $3.0(3.0-3.0)$ & $3.0(2.0-5.0)$ & $3.0(3.0-3.0)$ \\
\hline MMSE atención-cálculo & $5.0(2.0-5.0)$ & $5.0(0.0-5.0)^{p}$ & $3.0(0.0-5.0)^{p}$ \\
\hline MMSE recuerdo diferido & $2.0(1.0-3.0)$ & $2.0(0.0-3.0)$ & $2.0(1.0-2.0)$ \\
\hline MMSE lenguaje & $9.0(9.0-9.0)^{a, r}$ & $9.0(5.0-9.0)^{q . s}$ & $7.0(6.0-9.0)^{r, s}$ \\
\hline MMSE total & $29.0(26.0-30.0)^{t}$ & $28.0(19.0-30.0)^{u}$ & $26.0(21.0-26.0)^{\mathrm{tu} u}$ \\
\hline
\end{tabular}

$a=p<0.001, b=p<0.004, c=p<0.001, d=p<0.018, e=p<0.001, f=p<0.003, g=p<0.028, h=p<0.018, i=p<0.012, j=p<0.18, k=p<0.001, l=p<0.001, m=p<0.017$,

$n=p<0.04,0=p<0.054, p=p<0.022, q=p<0.038, r=p<0.026, s=p<0.029, t=p<0.018, u=p<0.013$

*Los resultados expresan medianas (mínimos y máximos).

MMSE: Mini Mental Scale Examination; SARCF: cuestionario para diagnóstico rápido de sarcopenia.

adultos mayores tienen deficiencia de $25(\mathrm{OH}) \mathrm{D}^{16,36,37}$. Sin embargo, es importante mencionar que quizás no se obtuvo información precisa sobre el consumo de multivitamínicos.
No se ha definido la concentración óptima de $25(\mathrm{OH}) \mathrm{D}$, pero se ha recomendado que sea $>30 \mathrm{ng} / \mathrm{dl}$, considerando insuficientes aquellas entre $20-30 \mathrm{ng} / \mathrm{dl}$. En este estudio, la mayoría de las participantes se 
Tabla 4. Valores obtenidos en marcadores de masa muscular, grasa visceral, desempeño funcional, vitamina D y MMSE en 99 pacientes posmenopáusicas mayores de 50 años divididas según la edad

\begin{tabular}{|c|c|c|c|c|}
\hline & $50 \mathrm{a}<60$ años & $60 \mathrm{a}<70$ años & $70 \mathrm{a}<80$ años & $\geq 80$ años \\
\hline Edad (años) & $55.0(50.0-59.0)^{a, b, c}$ & $64.0(60.0-69.0)^{\mathrm{a}, \mathrm{d}, \mathrm{e}}$ & $72.0(70.0-76.0)^{\mathrm{b}, \mathrm{d}, \mathrm{f}}$ & $85.5(80.0-90.0)^{c, e, f}$ \\
\hline Peso (kg) & $65.0(48.6-99.5)$ & $69.5(50.70-90.0)^{9}$ & $59.0(46.0-81.0)^{9}$ & $57.0(43.10-82.0)$ \\
\hline Talla (m) & $1.58(1.43-1.68)^{\mathrm{h}}$ & $1.55(1.45-1.70)^{i}$ & $1.50(1.42-1.60)^{h, i}$ & $1.49(1.40-1.69)$ \\
\hline Índice de masa corporal (kg/m²) & $27.08(19.9-39.8)$ & $28.7(20.8-36.3)$ & $26.0(21.6-34.2)$ & $29.9(18.7-41.8)$ \\
\hline Perímetro de la cintura $(\mathrm{cm})$ & $27.1(19.9-39.8)^{i}$ & $92.0(66.0-105.0)^{j}$ & $88.0(74.0-97.0)$ & $96.5(73.0-108.0)$ \\
\hline Perímetro de la cadera $(\mathrm{cm})$ & $99.0(78.0-132.0)^{k}$ & $105.8(89.0-118.0)^{k}$ & $102.0(82.0-116.0)$ & $107.5(86.0-113.0)$ \\
\hline Índice cintura cadera & $0.84(0.75-0.99)$ & $0.87(0.69-0.97)$ & $0.86(0.79-0.93)$ & $0.88(0.76-0.96)$ \\
\hline Presión arterial sistólica (mm-Hg) & $120(100-140)^{!, m}$ & $122.5(100-140)^{\prime}$ & $120.0(100-140)$ & $125.0(120-150)^{\mathrm{m}}$ \\
\hline Presión arterial diastólica (mm-Hg) & $70(60-90)$ & $80(60-90)$ & $80(70-90)$ & $80(50-90)$ \\
\hline Área muscular de brazo $\left(\mathrm{cm}^{2}\right)$ & $29.8(16.8-48.6)$ & $30.7(16.7-46.8)$ & $26.6(17.4-68.3)$ & $27.6(16.6-35.9)$ \\
\hline $\mathrm{AMBLH}\left(\mathrm{cm}^{2}\right)$ & $23.4(10.3-42.1)$ & $24.2(10.2-40.3)$ & $20.1(10.9-61.8)$ & $20.1(10.1-29.4)$ \\
\hline Masa muscular total $(\mathrm{kg})$ & $17.5(11.9-26.1)$ & $18.0(11.7-26.3)^{\mathrm{n} . o}$ & $15.5(11.5-34.5)^{\mathrm{n}}$ & $15.8(10.6-21.1)^{\circ}$ \\
\hline Vitamina D (ng/ml) & $16.1(3.5-23.1)^{\mathrm{p}, \mathrm{q}}$ & $16.2(9.7-29.7)^{r . s}$ & $21.6(13.3-30.1)^{p, r, t}$ & $26.7(16.0-37.1)^{q, s, t}$ \\
\hline Densidad corporal & $1.007(0.994-1.034)$ & $1.008(0.994-1.029)$ & $1.007(0.999-1.030)$ & $1.008(0.996-1.020)$ \\
\hline Porcentaje de grasa (\%) & $41.0(28.6-47.2)$ & $42.4(26.6-48.1)$ & $41.0(35.0-45.0)$ & $38.7(30.3-46.8)$ \\
\hline Promedio de fuerza de agarre $(\mathrm{kg})$ & $22.4(8.0-39.7)^{\mathrm{u}, v}$ & $20.5(11.1-31.6)^{w, x}$ & $16.7(11.7-29.3)^{u, w, y}$ & $10.7(4.1-16.8)^{v, x, y}$ \\
\hline $\begin{array}{l}\text { Puntuación de la prueba corta de } \\
\text { desempeño físico }\end{array}$ & $12.0(7.0-12.0)^{\mathrm{z}, \mathrm{aa}, \mathrm{bb}}$ & $11.6(5.0-12.0)^{z, c c}$ & $10.0(5.0 .-12.0)^{a a, d d}$ & $4.5(3.0-10.0)^{b b, c c, d d}$ \\
\hline Grasa subcutánea (cm) & $2.0(0.9-5.3)$ & $2.1(0.7-3.8)$ & $2.0(0.9-4.4)$ & $2.1(0.8-3.8)$ \\
\hline Grasa visceral (cm) & $3.6(1.5-8.9)$ & $4.6(1.6-6.8)$ & $4.1(1.7-7.8)$ & $3.6(1.4-8.5)$ \\
\hline Grasa visceral/grasa subcutánea & $1.8(0.9-5.0)$ & $2.2(1.1-5.8)$ & $1.9(1.2-3.7)$ & $1.5(0.8-5.0)$ \\
\hline SARC-F total & $0.0(0.0-7.0)^{e e, f f}$ & $1.0(0.0-4.0)^{g 9}$ & $2.0(0.0-9.0)^{e e, h h}$ & $8.0(3.0-11.0)^{f f, g g, h h}$ \\
\hline MMSE orientación temporal & $5.0(4.0-5.0)^{i i}$ & $5.0(3.0-5.0)$ & $5.0(3.0-5.0)^{\mathrm{nn}}$ & $5.0(2.0-5.0)$ \\
\hline MMSE orientación espacial & $5.0(3.0-6.0)^{i j}$ & $5.0(3.0-5.0)$ & $5.0(3.0-5.0)$ & $4.5(4.0-5.0)^{j i}$ \\
\hline MMSE recuerdo inmediato & $1.0(1.0-1.0)$ & $1.0(1.0-2.0)$ & $1.0(1.0-2.0)$ & $1.0(1.0-1.0)$ \\
\hline MMSE fijación & $3.0(3.0-5.0)$ & $3.0(3.0-3.0)$ & $3.0(2.0-5.0)$ & $3.0(3.0-3.0)$ \\
\hline MMSE atención-cálculo & $5.0(0.0-5.0)^{\mathrm{kk}}$ & $5.0(3.0-5.0)^{\| \prime}$ & $4.0(0.0-5.0)^{k \mathrm{k}, \|}$ & $5.0(0.0-5.0)$ \\
\hline MMSE recuerdo diferido & $2.0(1.0-3.0)$ & $2.0(1.0-3.0)$ & $2.0(0.0-3.0)$ & $2.0(1.0-3.0)$ \\
\hline MMSE lenguaje & $9.0(5.0-9.0)^{\mathrm{mm}, \mathrm{nn}}$ & $9.0(7.0-9.0)^{\circ o, p p}$ & $8.0(5.0-9.0)^{\mathrm{mm}, 00}$ & $7.0(6.0-9.0)^{n n, p p}$ \\
\hline MMSE total & $28.0(21.0-30.0)^{q q, r r}$ & $29.0(24.0-30.0)^{\text {ss.tt }}$ & $26.0(19.0-30.0)^{r, s s}$ & $25.5(21.0-28.0)^{r, t, t t}$ \\
\hline \multicolumn{5}{|c|}{ 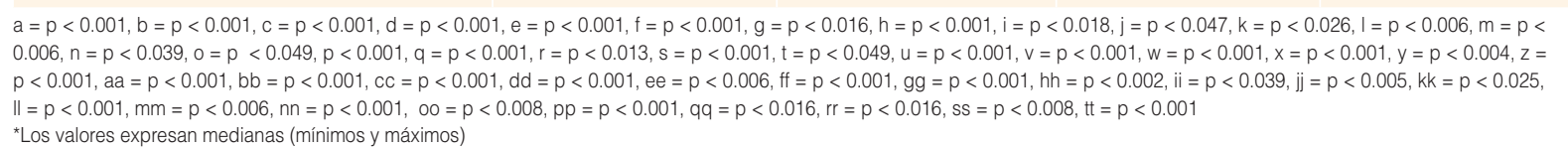 } \\
\hline
\end{tabular}

encontraban en un rango de insuficiencia similar al informado en las poblaciones de otros estudios ${ }^{36,37}$.

En este estudio, aquellas con IMC más alto tuvieron concentraciones más altas de $25(\mathrm{OH}) \mathrm{D}$, contrario a lo reportado en la literatura ${ }^{18,38}$ y un puntaje MMSE mayor, similar a lo ya reportado ${ }^{39}$.

La función cognitiva se vio afectada negativamente por la edad como en otros informes ${ }^{21}$, y también por 
Tabla 5. Valores obtenidos en marcadores de masa muscular, grasa visceral, desempeño funcional, vitamina D y MMSE en 99 pacientes posmenopáusicas mayores de 50 años divididas según el índice de masa corporal y el índice cintura: cadera

\begin{tabular}{|c|c|c|c|c|}
\hline & $I M C \leq 27 \mathrm{~kg} / \mathrm{m}^{2}$ & IMC> 27 kg/m² & $I C C \leq 0.85$ & ICC $>0.85$ \\
\hline Edad (años) & $60.0(50.0-90.0)$ & $61.0(50.0-90.0)$ & $59.5(50.0-85.0)$ & $62.0(50.0-90.0)$ \\
\hline Peso (kg) & $56.7(43.1-73.0)^{\text {a }}$ & $73.5(56.0-99.5)^{\text {a }}$ & $62.8(43.1-90.0)$ & $68.3(48.60-99.5)$ \\
\hline Talla (m) & $1.55(1.42-1.69)$ & $1.54(1.40-1.70)$ & $1.56(1.42-1.70)$ & $1.54(1.40-1.68)$ \\
\hline Índice de masa corporal (Kg/m²) & $24.2(18.7-26.8)^{b}$ & $30.4(27.0-41.8)^{b}$ & $26.3(18.7-34.6)^{c}$ & $28.4(19.9-41.8)^{c}$ \\
\hline Perímetro de la cintura $(\mathrm{cm})$ & $78.5(66.0-100.0)^{d}$ & $92.0(73.0-120.0)^{d}$ & $80.5(66.0-98.0)^{\mathrm{e}}$ & $92.5(71.0-120.0)^{e}$ \\
\hline Perímetro de la cadera $(\mathrm{cm})$ & $93.0(78.0-113.0)^{f}$ & $107.5(92.0-132.0)^{\dagger}$ & $100.5(82.0-118.0)$ & $105.0(78.0-132.0)$ \\
\hline Índice cintura: cadera & $0.84(0.73-0.99)$ & $0.87(0.69-0.98)$ & $0.81(0.69-0.85)^{g}$ & $0.89(0.86-0.99)^{g}$ \\
\hline Presión arterial sistólica (mmHg) & $120(100-150)^{g}$ & $120(95-140)^{g}$ & $120(100-140)^{h}$ & $120(95-150)^{h}$ \\
\hline Presión arterial diastólica (mmHg) & $70(50-90)^{i}$ & $80(60-90)^{i}$ & $70(50-90)^{i}$ & $80(60-90)^{j}$ \\
\hline Vitamina D (ng/ml) & $16.0(6.1-30.4)^{k}$ & $18.0(3.5-37.1)^{k}$ & $18.2(6.5-30.4)$ & $16.4(3.5-37.1)$ \\
\hline Área muscular de brazo $\left(\mathrm{cm}^{2}\right)$ & $28.4(16.3-67.4)^{\prime}$ & $28.5(17.6-50.9)^{\prime}$ & $28.7(16.4-67.4)^{m}$ & $28.0(16.3-50.9)^{m}$ \\
\hline AMBLH $\left(\mathrm{cm}^{2}\right)$ & $22.2(10.1-61.8)^{n}$ & $22.4(11.3-45.1)^{n}$ & $22.6(10.2-61.8)^{\circ}$ & $21.8(10.1-45.1)^{\circ}$ \\
\hline Masa muscular total (kg) & $12.3(7.6-17.1)^{p}$ & $14.0(6.8-30.2)^{p}$ & $12.4(7.6-19.0)$ & $13.6(6.8-30.2)$ \\
\hline Densidad corporal & $1.008(0.994-1.039)^{a}$ & $1.007(0.995-1.034)^{a}$ & $1.006(0.994-1.039)^{r}$ & $1.008(0.995-1.031)^{r}$ \\
\hline Porcentaje de grasa (\%) & $41.0(26.6-48.1)^{\mathrm{s}}$ & $41.7(28.6-47.2)^{\mathrm{s}}$ & $41.8(26.6-47.0)^{t}$ & $41.0(30.3-47.2)^{t}$ \\
\hline Promedio de fuerza de agarre $(\mathrm{kg})$ & $19.9(4.1-29.7)$ & $11.0(8.0-39.6)$ & $21.0(4.1-39.6)^{u}$ & $17.9(8.0-31.6)^{u}$ \\
\hline $\begin{array}{l}\text { Puntuación de la prueba corta de } \\
\text { desempeño físico }\end{array}$ & $12.0(2.0-12.0)^{\vee}$ & $10.5(2.0-12.0)^{\vee}$ & $12.0(4.0-12.0)^{w}$ & $11.0(3.0-12.0)^{w}$ \\
\hline Grasa subcutánea (cm) & $1.6(0.7-3.8)^{x}$ & $2.6(1.1-5.3)^{x}$ & $2.0(0.8-4.4)$ & $2.2(0.7-5.3)$ \\
\hline Grasa visceral (cm) & $3.1(1.4-8.5)^{y}$ & $5.1(1.5-8.9)^{y}$ & $3.6(1.4-7.2)^{z}$ & $4.9(1.6-8.9)^{z}$ \\
\hline Grasa visceral/grasa subcutánea & $2.0(1.1-5.0)$ & $1.8(0.8-5.8)$ & $1.8(0.9-5.0)$ & $2.1(0.8-5.8)$ \\
\hline SARC-F total & $1.0(0.0-11.0)$ & $1.00(0.0-9.0)$ & $1.0(0.0-10.0)$ & $1.0(0.0-11.0)$ \\
\hline MMSE orientación temporal & $5.0(2.0-5.0)^{\mathrm{aa}}$ & $5.0(4.0-5.0)^{\mathrm{aa}}$ & $5.0(3.0-5.0)$ & $5.0(3.0-5.0)$ \\
\hline MMSE orientación espacial & $5.0(3.0-6.0)$ & $5.0(3.0-5.0)$ & $5.0(3.0-5.0)$ & $5.0(3.0-5.0)$ \\
\hline MMSE recuerdo inmediato & $1.0(1.0-2.0)$ & $1.0(1.0-2.0)$ & $1.0(1.0-2-0)$ & $1.0(1.0-2.0)$ \\
\hline MMSE fijación & $3.0(2.0-5.0)$ & $3.0(2.0-3.0)$ & $3.0(2.0-5.0)$ & $3.0(2.0-5.0)$ \\
\hline MMSE atención-cálculo & $5.0(0.0-5.0)$ & $5.0(3.0-5.0)$ & $5.0(0.0-5.0)$ & $5.0(0.0-5.0)$ \\
\hline MMSE recuerdo diferido & $2.0(0.0-3.0)$ & $2.0(0.0-3.0)$ & $2.0(0.0-3.0)$ & $2.0(0.0-3.0)$ \\
\hline MMSE lenguaje & $9.0(5.0-9.0)$ & $9.0(5.0-9.0)$ & $9.0(5.0-9.0)$ & $9.0(5.0-9.0)$ \\
\hline MMSE total & $28.0(19.0-30.0)^{b b}$ & $29.0(24.0-30.0)^{\mathrm{bb}}$ & $28.0(19.0-30.0)$ & $28.0(19.0-30.0)$ \\
\hline
\end{tabular}

$a=p<0.001, b=p<0.001 c=p<0.004 d=p<0.001 e=p<0.001, f=p<0.001, g=p<0.001, h=p<0.007, i=p<0.004, j=p<0.041, k=p<0.023, I=p<0.023, m=$

$p<0.036, n=p<0.023,0=p<0.036, p=p<0.03, q=p<0.023, r=p<0.001, s=p<0.02, t=p<0.001, u=p<0.02, v=p<0.048, w=p<0.003, x=p<0.024, y=p<0.001$,

$z=p<0.012, a a=p<0.001, b b=p<0.011$

*Los valores expresan medianas (mínimos y máximos).

AMBLH: Área muscular de brazo libre de hueso; ICC: Índice cintura cadera; IMC: Índice de masa corporal; MMSE: Mini Mental Scale Examination; SARC-F: cuestionario para diagnóstico rápido de sarcopenia;

las concentraciones altas de $25(\mathrm{OH}) \mathrm{D}$ contrario a lo reportado 23,31 . Por lo anterior es posible concluir que las concentraciones elevadas de $25(\mathrm{OH}) \mathrm{D}$, se asocian con una puntuación más baja en las pruebas de desempeño físico y del MMSE.

\section{Financiamiento}

La presente investigación no recibió ninguna beca específica de agencias de los sectores públicos, comercial o sin ánimo de lucro. 
Gaceta Médica de México. 2021;157

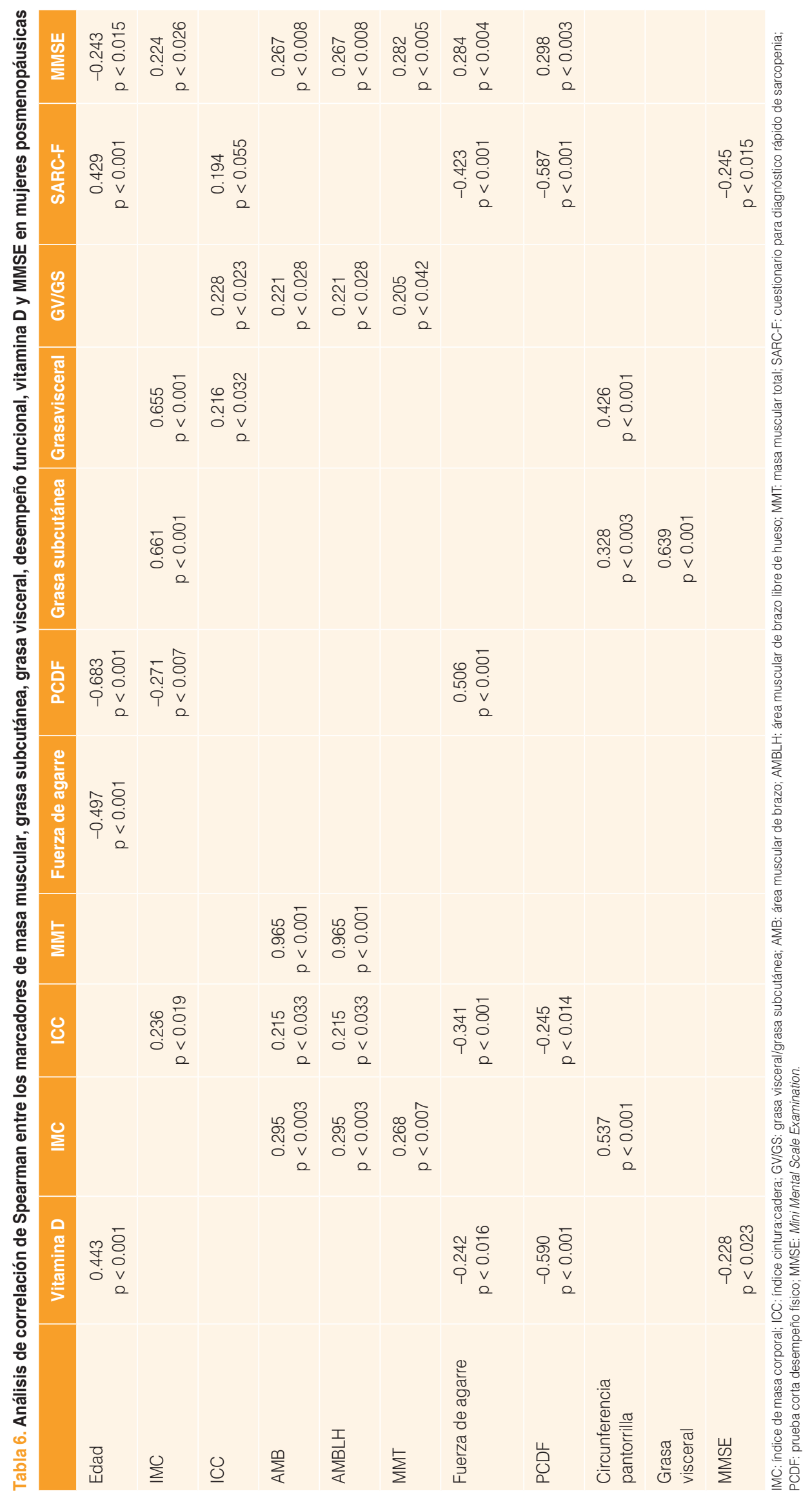

510 


\section{Conflicto de intereses}

Los autores declaran no tener conflicto de intereses alguno.

\section{Agradecimientos}

Los autores agradecen al Dr. Miguel Ángel López Valle por la derivación de pacientes.

\section{Responsabilidades éticas}

Protección de personas y animales. Los autores declaran que para esta investigación no se han realizado experimentos en seres humanos ni en animales.

Confidencialidad de los datos. Los autores declaran que han seguido los protocolos de su centro de trabajo sobre la publicación de datos de pacientes.

Derecho a la privacidad y consentimiento informado. Los autores han obtenido el consentimiento informado de los pacientes y/o sujetos referidos en el artículo. Este documento obra en poder del autor de correspondencia.

\section{Bibliografía}

1. Suetta C, Haddock B, Alcazar J, Noerst T, Hansen OM, Ludvig H, et al. The Copenhagen Sarcopenia Study: lean mass, strength, power, and physical function in a Danish cohort aged 20-93 years. J Cachexia Sarcopenia Muscle. 2019;10(6):1316-29.

2. Cruz-Jentoft AJ, Baeyens JP, Bauer JM, Boirie Y, Cederholm T, Landi F, et al. Sarcopenia: European consensus on definition and diagnosis. Age Ageing. 2010;39(4):412-23.

3. Cruz-Jentoft AJ, Bahat G, Bauer J, Boirie $Y$, Bruyère $O$, Cederholm T, et al. Sarcopenia: revised European consensus on definition and diagnosis. Age Ageing. 2019;48(1):16-31

4. Fielding RA, Vellas B, Evans WJ, Bhasin S, Morley JE, Newman AB, et al. Sarcopenia: an undiagnosed condition in older adults. Current consensus definition: prevalence, etiology, and consequences. International Working Group on Sarcopenia. J Am Med Dir Assoc. 2011;12(4):249-56.

5. Landi F, Liperoti R, Russo A, Giovannini S, Tosato M, Capoluongo E, et al. Sarcopenia as a risk factor for falls in elderly individuals: Results from the iISIRENTE study. Clin Nutr. 2012;31(5):652-8.

6. Marty E, Liu Y, Samuel A, Or O, Lane J. A review of sarcopenia: Enhancing awareness of an increasingly prevalent disease. Bone. 2017;105:276-86.

7. Mijnarends DM, Meijers JMM, Halfens RJG, Ter Borg S, Luiking YC, Verlaan $\mathrm{S}$, et al. Validity and reliability of tools to measure muscle mass, strength, and physical performance in community-dwelling older people: A systematic Review. J Am Med Dir Assoc. 2013;14(3):170-8.

8. Morley JE, Cao L. Rapid screening for sarcopenia. J Cachexia Sarcopenia Muscle. 2015;6(4):312-4.

9. Bahat G, Yilmaz O, Kılıç C, Oren MM, Karan MA. Performance of SARC-F in regard to sarcopenia definitions, muscle mass and functional measures. J Nutr Health Aging. 2018:22(8):898-903.

10. Leong DP, Teo KK, Rangarajan S, Kutty VR, Lanas F, Hui C, et al. Reference ranges of handgrip strength from 125,462 healthy adults in 21 countries: a prospective urban rural epidemiologic (PURE) study. J Cachexia Sarcopenia Muscle. 2016;7(5):535-46.

11. Yoo J II, Choi H, Ha YC. Mean hand grip strength and cut-off value for sarcopenia in Korean adults using KNHANES VI. J Korean Med Sci. 2017;32(5):868-72

12. McCarthy EK, Horvat MA, Holtsberg PA, Wisenbaker JM. Repeated chair stands as a measure of lower limb strength in sexagenarian women. J Gerontol A Biol Sci Med Sci. 2004;59(11):1207-12.

13. Evans WJ, Bhasin S, Cress E, Espeland MA, Ferrucci L, Fried LP, et al. Functional outcomes for clinical trials in frail older persons: Time to be moving. J Gerontol A Biol Sci Med Sci. 2008;63(2):160-4.
14. Freire AN, Guerra RO, Alvarado B, Guralnik JM, Zunzunegui MV. Validity and reliability of the short physical performance battery in two diverse older adult populations in Quebec and Brazil. J Aging Health. 2012;24(5):863-78.

15. Guralnik JM, Simonsick EM, Ferrucci L, Glynn RJ, Berkman LF, Blazer DG, et al. A short physical performance battery assessing lower extremity function: Association with self-reported disability and prediction of mortality and nursing home admission. J Gerontol. 1994;49(2):M85-M94.

16. Conzade R, Grill E, Bischoff-Ferrari HA, Ferrari U, Horsch A, Koenig W, et al. Vitamin $D$ in relation to incident sarcopenia and changes in muscle parameters among older adults: The KORA-Age Study. Calcif Tissue Int. 2019;105(2):173-82.

17. Liu G, Lu L, Sun Q, Ye X, Sun L, Liu X, et al. Poor vitamin D status is prospectively associated with greater muscle mass loss in middle-aged and elderly chinese individuals. J Acad Nutr Diet. 2014;114(10):1544-1551.e2.

18. Mathieu SV, Fischer K, Dawson-Hughes B, Freystaetter G, Beuschlein F, Schietzel S, et al. Association between 25-hydroxyvitamin D status and components of body composition and glucose metabolism in older men and women. Nutrients. 2018;10(12)

19. Carranza-Lira S, Bárcena-Jacobo TD, Sandoval-Barragán MP, Ramos-León JC. Visceral adiposity after tibolone use. Int J Gynaecol Obstet. 2011;115(2):191-3.

20. Blümel JE, Salinas C, Danckers L, Tserota K, Ojeda E, Vallejo MS, et al. Muscle health in Hispanic women. REDLINC VIII. Climacteric. 2020;23(2):184-91.

21. Carranza-Lira $S$, Carpio-Bárcenas $P$, Carranza-Lira $S$, Carpio-Bárcenas $P$ Tiempo trascurrido a partir de la menopausia y su repercusión en el deterioro cognitivo. Ginecol Obstet Mex. 2018;86(5):289-96.

22. Aveleyra E, Carranza-Lira S, Ulloa-Aguirre A, Ostrosky-Solís F. Cognitive effects of hormone therapy in early postmenopausal women. Int $J$ Psychol. 2005;40(5):314-23.

23. Laughlin GA, Kritz-Silverstein D, Bergstrom J, Reas ET, Jassal SK Barrett-Connor $\mathrm{E}$, et al. Vitamin $\mathrm{D}$ insufficiency and cognitive function trajectories in older adults: The Rancho Bernardo Study. J Alzheimers Dis. 2017; 58(3):871-83.

24. Chei CL, Raman $P$, Yin ZX, Shi XM, Zeng Y, Matchar DB. Vitamin D levels and cognition in elderly adults in China. J Am Geriatr Soc. 2014;62(11):2125-9.

25. Balion C, Griffith LE, Strifler L, Henderson M, Patterson C, Heckman G, et al. Vitamin $D$, cognition, and dementia: a systematic review and meta-analysis. Neurology. 2012;79(13):1397-405.

26. Jorde R, Mathiesen EB, Rogne S, Wilsgaard T, Kjærgaard M, Grimnes G, et al. Vitamin D and cognitive function: The Tromsø Study. J Neurol Sci. 2015;355(1-2):155-61.

27. Llewellyn DJ, Lang IA, Langa KM, Muniz-Terrera G, Phillips CL, Cherubini $A$, et al. Vitamin $D$ and risk of cognitive decline in elderly persons. Arch Intern Med. 2010;170(13):1135-41.

28. Peterson A, Mattek N, Clemons A, Bowman GL, Buracchio T, Kaye J, et al. Serum vitamin $D$ concentrations are associated with falling and cognitive function in older adults. J Nutr Health Aging. 2012;16(10):898-901.

29. Navarrete-Reyes AP, García-Muñoz I, García-Lara JMA, Torres-Carrillo $\mathrm{NM}$, Amieva H, Avila-Funes JA. 25-OH-vitamin D Is not associated with cognitive performance among Mexican community-dwelling older persons. J Frailty Aging. 2015;4(2):74-9.

30. Olsson E, Byberg L, Karlström B, Cederholm T, Melhus H, Sjögren P, et al. Vitamin $D$ is not associated with incident dementia or cognitive impairment: an 18-y follow-up study in community-living old men. Am J Clin Nutr. 2017;105(4):936-43.

31. Folstein MF, Folstein SE, McHugh PR. "Mini-Mental State". A practical method for grading the cognitive state of patients for the clinician. J Psychiatr Res. 1975;12(3):189-98

32. Llamas Velasco S, Llorente Ayuso L, Contador I, Bermejo Pareja F. Versiones en español del Minimental State Examination (MMSE). Cuestiones para su uso en la práctica clínica. Rev Neurol. 2015;61(08):363-71.

33. Composición corporal y medidas antropométricas [Internet]. UNAM, Facultad de Medicina, Departamento de Fisiología; 2016. Disponible en: http://fisiologia.facmed.unam.mx/wp-content/uploads/2019/02/1-PracticaComposicionCorporal.pdf

34. Carranza-Lira S, Muro AMF, Ortiz SR. Relation of skinfold thickness and visceral fat with the endothelial function in Mexican postmenopausal women. Prz Menopauzalny. 2015;14(2):90-6.

35. Fried LP, Tangen CM, Walston J, Newman AB, Hirsch C, Gottdiener J, et al. Fraility in older adults: evidence for a phenotype. J Gerontol A Biol Sci Med Sci. 2001;56(3):M146-56.

36. Garg MK, Sandeep K, Brar KS, Rakesh M. Vitamin d deficiency in elderly: implications, prevention and treatment. J Ind Acad Geriatr. 2012;8:77-92.

37. Boettger SF, Angersbach B, Klimek CN, Monteiro Wanderley AL, Shaibekov $A$, Sieske $L$, et al. Prevalence and predictors of vitamin $D$ deficiency in frail older hospitalized patients. BMC Geriatrics. 2018;18:219.

38. Rafiq S, Jeppesen PB. Body mass index, vitamin D, and type 2 diabetes: a systematic review and meta-analysis. Nutrients. 2018;10(9):1182.

39. Cronk BB, Johnson DK, Burns JM. Alzheimer's disease neuroimaging initiative. Body mass index and cognitive decline in mild cognitive impairment. Alzheimer Dis Assoc. 2010;24(2):126-30. 